\title{
A study on normal multi-fuzzy BG-ideals of BG-algebra
}

\author{
R. Muthuraj ${ }^{1}$ and S. Devi ${ }^{2 *}$
}

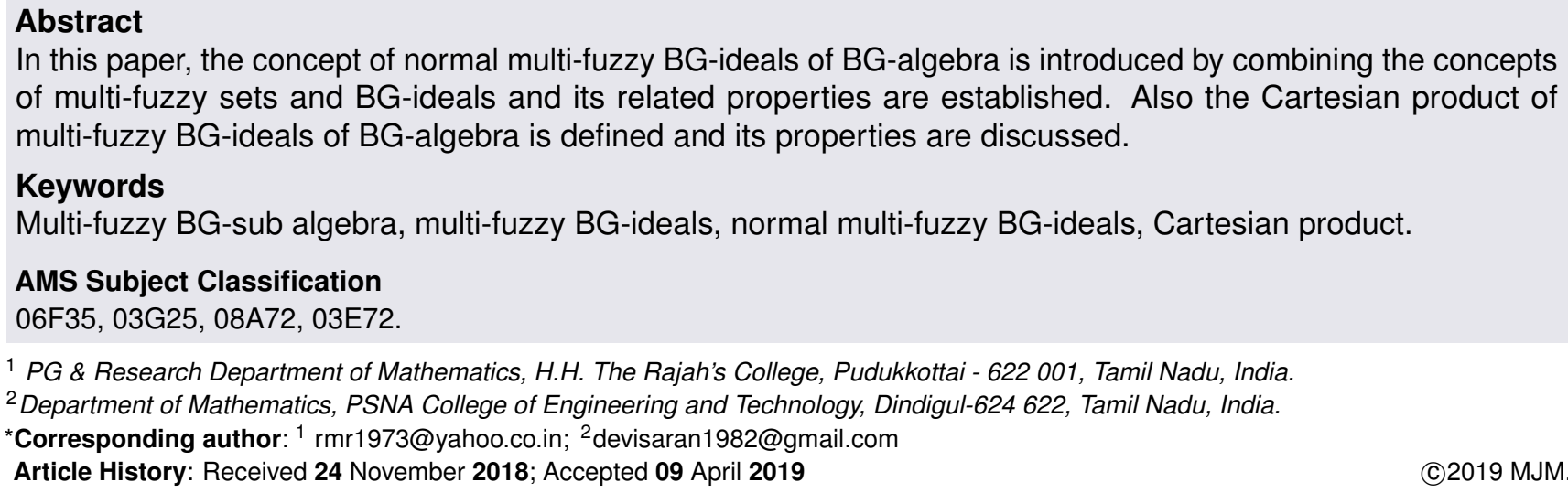

${ }^{1}$ PG \& Research Department of Mathematics, H.H. The Rajah's College, Pudukkottai - 622 001, Tamil Nadu, India.

2 Department of Mathematics, PSNA College of Engineering and Technology, Dindigul-624 622, Tamil Nadu, India.

*Corresponding author: ${ }^{1}$ rmr1973@yahoo.co.in; ${ }^{2}$ devisaran1982@gmail.com

Article History: Received 24 November 2018; Accepted 09 April 2019

\section{Contents}

1 Introduction ................................ 394

2 Preliminaries .............................. 394

3 Normal multi-fuzzy BG-ideals of BG-algebra ..... 395

4 Cartesian product of multi-fuzzy BG-ideals ...... 396

4.1 Corollary . . . . . . . . . . . . . . . . . . 397

5 Conclusion ................................. 398

References ............................... 398

\section{Introduction}

As the extension of fuzzy sets[20], the concept of multifuzzy set that is, a set with multi-membership values was introduced by S.Sabu and T.V.Ramakrishnan[17],[18] in 2000. Many researchers applied the concept of fuzzy sets in various abstract algebras and presented some theorems. At first, the two main abstract algebras: BCK-algebras and BCI-algebras were initiated by Y.Imai and K.Iseki[4-6]. It is shown that the class of BCK-algebras is a proper subclass of the class of BCIalgebras. J.Neggers and H.S.Kim[14]introduced a new notion, called a B-algebra. In 2005, C.B.Kim and H.S.Kim[7] introduced the notion of a BG-algebra which is a generalization of B-algebras. S.S.Ahn and H.D.Lee[3] fuzzified this BGalgebra and discussed some properties.The concept of fuzzy ideals of BG-algebra was presented by R.Muthuraj et.al[8] in 2010 and it is normalised by A.Prasanna et.al[16]. By applying the concept of multi-fuzzy sets in BG-algebra and BG-ideal, R.Muthuraj and S.Devi[9, 13] defined the notion of multi-fuzzy sub algebra and multi-fuzzy BG-ideals of BGalgebra and discussed the properties based on level sets. In this paper, we extend these results to the normal multi-fuzzy BG-ideals and also study some of its related properties. Also the Cartesian product of multi-fuzzy BG-ideals is defined.

\section{Preliminaries}

In this section, some fundamental definitions are given which will be used in the sequel.

Definition 2.1. [7] A BG-algebra is a non empty set $X$ with a constant 0 and a binary operation "*" statisfying the following axioms:
(1) $x * x=0$
(2) $x * 0=x$
(3) $(x * y) *(0 * y)=x$, forall $x, y \in X$.

Example 2.2. [7] Let $X=\{0,1,2\}$ be a set with the following table:

Then $(X ; *, O)$ is a BG-algebra.

Definition 2.3. [17] Let $X$ be a non-empty set and let $\left\{L_{i}\right.$ : $i \in P\}$ be a family of complete lattices. A multi-fuzzy set $A$ in $X$ is a set of ordered sequences: 


\begin{tabular}{|l|l|l|l|}
\hline$*$ & 0 & 1 & 2 \\
\hline 0 & 0 & 1 & 2 \\
\hline 1 & 1 & 0 & 1 \\
\hline 2 & 2 & 2 & 0 \\
\hline
\end{tabular}

$A=\left\{<x, \mu_{1}(x), \mu_{2}(x), \ldots \mu_{i}(x), \ldots>: x \in X\right\}$ where $\mu_{i} \in$ $L_{i}^{X}$, fori $\in P$.

\section{Remark 2.4. [17]}

If the sequences of the membership functions have only $k$-terms(finite number of terms), $k$ is called the dimension of $A$. If $L_{i}=[0,1]$ (for $\mathrm{i}=1,2, \ldots \mathrm{k}$ ), then the set of all multi-fuzzy sets in $\mathrm{X}$ of dimenstion $\mathrm{k}$ is denoted by $M^{k} F S(\mathrm{X})$. The multimebership function $\mu_{A}$ is a function from $\mathrm{X}$ to $I^{k}$ such that for all $\mathrm{x}$ in $\mathrm{X}, \mu_{A}(x)=\left(\mu_{1}(x), \mu_{2}(x), \ldots, \mu_{k}(x)\right)$.

For the sake of simplicity, we denote the multi-fuzzy set $A=\left\{\left(x, \mu_{1}(x), \mu_{2}(x), \ldots, \mu_{k}(x)\right): x \in X\right\}$

as $A=\left(\mu_{1}, \mu_{2}, \ldots \mu_{k}\right)$.

Definition 2.5. [17] Let $k$ be a positive integer and let $\mu$ and $v$ in $M^{k} F S(X)$, that is

$\mu=\left(\mu_{1}, \mu_{2}, \ldots \mu_{k}\right)=\left\{\left(x, \mu_{1}(x), \mu_{2}(x), \ldots \mu_{k}(x)\right): x \in X\right\}$ and $v=\left(v_{1}, v_{2}, \ldots v_{k}\right),=\left\{\left(x, v_{1}(x), v_{2}(x), \ldots v_{k}(x)\right): x \in X\right\}$ then we have the following relations and operations:

(1) $\mu \subseteq v$ if and only if $\mu_{i} \leq v_{i}$, for all $i=1,2, \ldots k$;

(2) $\mu=v$ if and only if $\mu_{i}=v_{i}$, for all $i=1,2, \ldots k$;

(3) $\mu \cup v=\left(\mu_{1} \cup v_{1}, \ldots \mu_{k} \cup v_{k}\right)$ $=\left\{\left(x, \max \left(\mu_{1}(x), v_{1}(x)\right), \ldots \max \left(\mu_{k}(x), v_{k}(x)\right)\right): x \in X\right\}$

(4) $\mu \cap v=\left(\mu_{1} \cap v_{1}, \ldots \mu_{k} \cap v_{k}\right)$ $=\left\{\left(x, \max \left(\mu_{1}(x), v_{1}(x)\right), \ldots \max \left(\mu_{k}(x), v_{k}(x)\right)\right): x \in X\right\}$

Definition 2.6. [9] Let $A$ be a multi-fuzzy set in a BG-algebra $X$. Then $A$ is called a multi-fuzzy subalgebra of $X$ if $A(x * y) \geq$ $\min \{A(x), A(y)\}, \forall x, y \in X$.

Definition 2.7. [13] Let $A$ be a multi-fuzzy set in X. Then A is called a multi-fuzzy $B G$-ideal in $X$ if it satisfies the following conditions:

$$
\begin{aligned}
& \text { i } A(0) \geq A(x) \\
& \text { ii. } A(x) \geq \min \{A(x * y), A(y)\} \\
& \text { iii. } A(x * y) \geq \min \{A(x), A(y)\}, \forall x, y \in X
\end{aligned}
$$

\section{Normal multi-fuzzy BG-ideals of BG-algebra}

In this section, normal multi-fuzzy BG-ideals of BGalgebra is defined and discuss some of its related properties.

Definition 3.1. A multi-fuzzy $B G$-ideal $A$ in $X$ is called normal, if there exists $x \in X$ such that $A(x)=1_{k}$ where $1_{k}=$ $(1,1,1, \ldots$ ktimes $)$.
Lemma 3.2. A multi-fuzzy BG-ideal $A$ in $X$ is normal if and only if $A(0)=1_{k}$.

Example 3.3. Let $X=\{0,1,2,3\}$ be the set with the following table

\begin{tabular}{|c|c|c|c|c|}
\hline$*$ & 0 & 1 & 2 & 3 \\
\hline 0 & 0 & 1 & 2 & 3 \\
\hline 1 & 1 & 0 & 3 & 2 \\
\hline 2 & 2 & 3 & 0 & 1 \\
\hline 3 & 3 & 2 & 1 & 0 \\
\hline
\end{tabular}

Then $(X, *, 0)$ is a BG-algebra. Define a multi-fuzzy set $A$ in $X$ by $A(0)=A(1)=(1,1,1)$ and $A(2)=A(3)=$ $(0.8,0.6,0.5)$. Clearly $A$ is a normal multi-fuzzy $B G$-ideal in $X$.

Theorem 3.4. Let $A$ be a MFS in a BG-algebra $X$. Then $A^{+}(x)=A(x)+1_{k}-A(0), \forall x \in X$ is a normal multi-fuzzy $B G$-ideal of $X$ which contains $A$.

Proof. Let $x, y \in X$

$$
\begin{gathered}
\mu_{i}^{+}(x)=\mu_{i}(x)+1-\mu_{i}(0) \\
\leq \mu_{i}(0)+1-\mu_{i}(0)=\mu_{i}^{+}(0) \forall i=1,2, . ., k \\
\min \left\{\mu_{i}^{+}(x * y), \mu_{i}^{+}(y)\right\}=\min \left\{\mu_{i}(x * y)+1-\mu_{i}(0),\right. \\
\left.\mu_{i}(y)+1-\mu_{i}(0)\right\} \\
=\min \left\{\mu_{i}(x * y), \mu_{i}(y)\right\}+1-\mu_{i}(0) \\
\min \left\{\mu_{i}^{+}(x), \mu_{i}^{+}(y)\right\}=\min \left\{\mu_{i}(x)+1-\mu_{i}(0),\right. \\
\left.\mu_{i}(y)+1-\mu_{i}(0)\right\} \\
=\mu_{i}(x)+1-\mu_{i}^{+}(x) \forall i=1,2, k \\
=\min \left\{\mu_{i}(x), \mu_{i}(y)\right\}+1-\mu_{i}(0) \\
\leq \mu_{i}(x * y)+1-\mu_{i}(0) \\
=\mu_{i}^{+}(x * y) \forall i=1,2, . ., k \\
=A(0)+1_{k}-A(0)=1
\end{gathered}
$$

Therefore $A^{+}$is a normal multi-fuzzy BG-ideal in $X$. Clearly $A \subseteq A^{+}$. Thus $A^{+}$is a normal multi-fuzzy BG-ideal which contains A.

Corollary 3.5. If there is an element $x \in X$ such that $A^{+}(x)=$ 0 , then $A(x)=0$.

Proof: Since $A \subseteq A^{+}, A(x)=0$.

Corollary 3.6. (i)If $A$ itself is normal then $A=A^{+}$. (ii) If $A$ is a multi-fuzzy $B G$-ideal in $X$ then $\left(A^{+}\right)^{+}=A^{+}$.

Theorem 3.7. Let $A$ and $B$ be two normal multi-fuzzy $B G$ ideals in $X$. If $A \subseteq B$ then $X_{A} \subseteq X_{B}$. 
Proof. Let $X_{A}=\{x \in X / A(x)=A(0)\}$ and $X_{B}=\{x \in X / B(x)$ $=B(0)\}$. Let $x \in X_{A}$.

Then $v_{i}(x) \geq \mu_{i}(x)=\mu_{i}(0)=1=v_{i}(0)$ for every $i=1,2, \ldots k$ and so $v_{i}(x)=v_{i}(0)$.

This implies $x \in X_{B}$. Hence $X_{A} \subseteq X_{B}$.

Theorem 3.8. Let $A$ be a multi-fuzzy $B G$-ideal in $X$. If there exists a multi-fuzzy $B G$-ideal $B$ of $X$ such that $B^{+} \subseteq A$, then $A$ is normal.

Proof. Since $B$ is a multi-fuzzy BG-ideal in $X, B^{+}$is a normal multi-fuzzy BG-ideal in $X$.

Then $B^{+}(0)=1_{k} \Longrightarrow v_{i}^{+}(0)=1$ for every $i$.

$B^{+} \subseteq A \Longrightarrow v_{i}^{+}(x) \leq \mu_{i}(x), \forall x \in X$ and $i=1,2 \ldots k$

$1=v_{i}^{+}(0) \leq \mu_{i}(0)$ for every $i$.

That is, $1_{k}=B^{+}(0) \leq A(0)$. Hence $A$ is normal.

Theorem 3.9. Let $A$ be a multi-fuzzy BG-ideal and let $f$ : $[0, A(0)] \rightarrow[0,1]$ be an increasing function. Then a multifuzzy set $A^{f}: X \rightarrow[0,1]$ by $A^{f}(x)=f(A(x)) \forall x \in X$ is a multifuzzy $B G$-ideal in $X$. Also if $f(A(0))=1_{k}$, then $A^{f}$ is normal.

Proof. Since $f$ is an increasing function,

$\mu_{i}^{f}(0)=f\left(\mu_{i}(0)\right) \geq f\left(\mu_{i}(x)\right)=\mu_{i}^{f}(x) \forall i$

$\Longrightarrow A^{f}(0) \geq A^{f}(x)$

we have

$$
\begin{aligned}
\mu_{i}^{f}(x) & =f\left(\mu_{i}(x)\right) \\
& \geq f\left(\min \left\{\mu_{i}(x * y), \mu_{i}(y)\right\}\right) \\
& =\min \left\{f\left(\mu_{i}(x * y)\right), f\left(\mu_{i}(y)\right)\right\} \\
& =\min \left\{\mu_{i}^{f}(x * y), \mu_{i}^{f}(y)\right\} \forall i .
\end{aligned}
$$

and

$$
\begin{aligned}
\mu_{i}^{f}(x * y) & =f\left(\mu_{i}(x * y)\right) \\
& \geq f\left(\min \left\{\mu_{i}(x), \mu_{i}(y)\right\}\right) \\
& =\min \left\{f\left(\mu_{i}(x)\right), f\left(\mu_{i}(y)\right)\right\} \\
& =\min \left\{\mu_{i}^{f}(x), \mu_{i}^{f}(y)\right\} \forall i .
\end{aligned}
$$

This implies $A^{f}$ is a multi-fuzzy BG-ideal in $X$. If $f(A(0))=$ $1_{k}, A^{f}(0)=1_{k}$. Therefore $A^{f}$ is normal.

\section{Cartesian product of multi-fuzzy BG-ideals}

In this section, the Cartesian product of multi-fuzzy BGideals of BG-algebra is defined and its properties are discussed.

Definition 4.1. Let $A$ and $B$ be two MFSs of dimension k.Then Cartesian product of $A$ and $B$ is defined by $(A \times B)\left(x_{1}, x_{2}\right)=\left\{\min \left\{\mu_{1}\left(x_{1}\right), v_{1}\left(x_{2}\right)\right\}, \min \left\{\mu_{2}\left(x_{1}\right), v_{2}\left(x_{2}\right)\right\} \ldots\right.$ $\left.\min \left\{\mu_{k}\left(x_{1}\right), v_{k}\left(x_{2}\right)\right\}\right\}$ where $\left(x_{1}, x_{2}\right) \in X \times X$.
Theorem 4.2. If $A$ and $B$ are two multi-fuzzy $B G$-ideals of $X$, then $A \times B$ is also a multi-fuzzy $B G$-ideal of $X \times X$.

Proof.

$$
\begin{aligned}
(A \times B)(0,0)= & \left\{\min \left\{\mu_{1}(0), v_{1}(0)\right\},\right. \\
& \min \left\{\mu_{2}(0), v_{2}(0)\right\}, \\
& \left.\ldots \min \left\{\mu_{k}(0), v_{k}(0)\right\}\right\} \\
\geq\left\{\min \left\{\mu_{1}\left(x_{1}\right), v_{1}\left(x_{2}\right)\right\},\right. & \\
& \min \left\{\mu_{2}\left(x_{1}\right), v_{2}\left(x_{2}\right)\right\}, \\
& \left.\ldots \min \left\{\mu_{k}\left(x_{1}\right), v_{k}\left(x_{2}\right)\right\}\right\} \\
= & (A \times B)\left(x_{1}, x_{2}\right)
\end{aligned}
$$

Let $\left(x_{1}, x_{2}\right)$ and $\left(y_{1}, y_{2}\right)$ in $X \times X$. Then

$$
\begin{aligned}
& (A \times B)\left(x_{1}, x_{2}\right)=\left\{\min \left\{\mu_{1}\left(x_{1}\right), v_{1}\left(x_{2}\right)\right\},\right. \\
& \min \left\{\mu_{2}\left(x_{1}\right), v_{2}\left(x_{2}\right)\right\} \ldots \\
& \left.\min \left\{\mu_{k}\left(x_{1}\right), v_{k}\left(x_{2}\right)\right\}\right\} \\
& \geq\left\{\operatorname { m i n } \left\{\min \left\{\mu_{1}\left(x_{1} * y_{1}\right), \mu_{1}\left(y_{1}\right)\right\},\right.\right. \\
& \left.\min \left\{v_{1}\left(x_{2} * y_{2}\right), v_{1}\left(y_{2}\right)\right\}\right\}, \\
& \min \left\{\min \left\{\mu_{2}\left(x_{1} * y_{1}\right), \mu_{2}\left(y_{1}\right)\right\}\right. \text {, } \\
& \left.\min \left\{v_{2}\left(x_{2} * y_{2}\right), v_{2}\left(y_{2}\right)\right\}\right\}, \ldots \\
& \min \left\{\min \left\{\mu_{k}\left(x_{1} * y_{1}\right), \mu_{k}\left(y_{1}\right)\right\}\right. \text {, } \\
& \left.\left.\min \left\{v_{k}\left(x_{2} * y_{2}\right), v_{k}\left(y_{2}\right)\right\}\right\}\right\} \\
& =\min \left\{\min \left\{\mu_{1}\left(x_{1} * y_{1}\right), v_{1}\left(x_{2} * y_{2}\right)\right\}\right. \text {, } \\
& \min \left\{\mu_{2}\left(x_{1} * y_{1}\right), v_{2}\left(x_{2} * y_{2}\right)\right\} \ldots \\
& \left.\min \left\{\mu_{k}\left(x_{1} * y_{1}\right), v_{k}\left(x_{2} * y_{2}\right)\right\}\right\}, \\
& \left\{\min \left\{\mu_{1}\left(y_{1}\right), v_{1}\left(y_{2}\right)\right\}\right. \text {, } \\
& \min \left\{\mu_{2}\left(y_{1}\right), v_{2}\left(y_{2}\right)\right\} \\
& \left., \ldots \min \left\{\mu_{k}\left(y_{1}\right), v_{k}\left(y_{2}\right)\right\}\right\} \\
& =\min \left\{(A \times B)\left(x_{1} * y_{1}, x_{2} * y_{2}\right)\right. \text {, } \\
& \left.(A \times B)\left(y_{1}, y_{2}\right)\right\} \\
& =\min \left\{(A \times B)\left(\left(x_{1}, x_{2}\right) *\left(y_{1}, y_{2}\right)\right)\right. \text {, } \\
& \left.(A \times B)\left(y_{1}, y_{2}\right)\right\}
\end{aligned}
$$

$$
\begin{aligned}
(A \times B)\left(\left(x_{1}, x_{2}\right) *\left(y_{1}, y_{2}\right)\right)=(A \times B)\left(\left(x_{1} * y_{1}\right),\right. & \left.\left(x_{2} * y_{2}\right)\right) \\
=\{ & \min \left\{\mu_{1}\left(x_{1} * y_{1}\right),\right. \\
& \left.v_{1}\left(x_{2} * y_{2}\right)\right\}, \\
& \min \left\{\mu_{2}\left(x_{1} * y_{1}\right),\right. \\
& \left.v_{2}\left(x_{2} * y_{2}\right)\right\} \ldots \\
& \min \left\{\mu_{k}\left(x_{1} * y_{1}\right),\right. \\
& \left.\left.v_{k}\left(x_{2} * y_{2}\right)\right\}\right\}
\end{aligned}
$$




$$
\begin{aligned}
\geq & \min \left\{\min \left\{\mu_{1}\left(x_{1}\right), \mu_{1}\left(y_{1}\right)\right\},\right. \\
& \left.\min \left\{v_{1}\left(x_{2}\right), v_{1}\left(y_{2}\right)\right\}\right\}, \\
& \min \left\{\mu_{2}\left(x_{1}\right), \mu_{2}\left(y_{1}\right)\right\}, \\
& \left.\min \left\{v_{2}\left(x_{2}\right), v_{2}\left(y_{2}\right)\right\}\right\}, \ldots \\
& \min \left\{\mu_{k}\left(x_{1}\right), \mu_{k}\left(y_{1}\right)\right\}, \\
& \left.\min \left\{v_{k}\left(x_{2}\right), v_{k}\left(y_{2}\right)\right\}\right\} \\
= & \min \left\{\min \left\{\mu_{1}\left(x_{1}\right), v_{1}\left(x_{2}\right)\right\},\right. \\
& \min \left\{\mu_{2}\left(x_{1}\right), v_{2}\left(x_{2}\right)\right\} \ldots \\
& \min \left\{\mu_{k}\left(x_{1}\right), v_{k}\left(x_{2}\right)\right\}, \\
& \min \left\{\mu_{1}\left(y_{1}\right), v_{1}\left(y_{2}\right)\right\}, \\
& \min \left\{\mu_{2}\left(y_{1}\right), v_{2}\left(y_{2}\right)\right\} \ldots \\
& \left.\min \left\{\mu_{k}\left(y_{1}\right), v_{k}\left(y_{2}\right)\right\}\right\} \\
= & \min \left\{(A \times B)\left(x_{1}, x_{2}\right),\right. \\
& \left.(A \times B)\left(y_{1}, y_{2}\right)\right\}
\end{aligned}
$$

Hence $A \times B$ is a multi-fuzzy BG-ideal in $X \times X$.

\subsection{Corollary}

The converse of the above theorem may not be true as given in the following example. Example: Let $X=\{0,1,2,3\}$ be a BG-algebra as in example 3.3. Let $r=\left(r_{1}, r_{2}, \ldots r_{k}\right)$ and $s=\left(s_{1}, s_{2}, \ldots s_{k}\right)$ where $r_{i}$ and $s_{i} \in[0,1]$ such that $r \leq s$. Define two multi-fuzzy sets $A$ and $B$ by $A(x)=r, B(x)=s$ if $x=0$ and $B(x)=1_{k}$ otherwise.

If $x \neq 0$, then

$$
\begin{array}{r}
(A \times B)(x, x)=\left\{\min \left\{\mu_{1}(x), v_{1}(x)\right\}, \min \left\{\mu_{2}(x), v_{2}(x)\right\}\right. \\
\left.\ldots \min \left\{\mu_{k}(x), v_{k}(x)\right\}\right\} \\
\left.=\left\{\min \left\{r_{1}, 1\right\}, \min \left\{r_{2}, 1\right\}, \ldots \min \left\{r_{k}, 1\right\}\right\}\right\} \\
=\left\{r_{1}, r_{2}, \ldots r_{k}\right\}=\{r\} \\
=r
\end{array}
$$

If $x=0$, then

$$
\begin{array}{r}
(A \times B)(x, x)=\left\{\min \left\{\mu_{1}(x), v_{1}(x)\right\}, \min \left\{\mu_{2}(x), v_{2}(x)\right\} \ldots\right. \\
\left.\min \left\{\mu_{k}(x), v_{k}(x)\right\}\right\} \\
=\left\{\left\{\min \left\{r_{1}, s_{1}\right\}, \min \left\{r_{2}, s_{2}\right\}, \ldots \min \left\{r_{k}, s_{k}\right\}\right\}\right. \\
=\left\{r_{1}, r_{2}, \ldots r_{k}\right\}=\{r\} \\
=r
\end{array}
$$

Hence $A \times B$ is a multi-fuzzy BG-ideal of $X \times X$. Clearly $A$ is a multi-fuzzy BG-ideal of $X$. But $B$ is not a multi-fuzzy BG-ideal of $X$ since for $x=0, B(0)=s \leq 1_{k}=B(x)$.

Theorem 4.3. Let $A$ and $B$ be two multi-fuzzy sets in a $B G$ algebra $X$ such that $A \times B$ is a multi-fuzzy $B G$-ideal of $X \times X$. Then

i. Either $A(0) \geq A(x)$ or $B(0) \geq B(x)$

ii. If $A(0) \geq A(x)$, then either $B(0) \geq B(x)$ or $B(0) \geq B(x)$.

iii. If $B(0) \geq B(x)$, then either $A(0) \geq A(x)$ or $A(0) \geq B(x)$.
Proof. Let $A(0)<A(x)$ and $B(0)<B(x)$. Then $\mu_{i}(0)<\mu_{i}(x)$ and $v_{i}(0)<v_{i}(x), \forall i=1,2, \ldots k$

$$
\begin{gathered}
(A \times B)\left(x_{1}, x_{2}\right)=\left\{\min \left\{\mu_{1}\left(x_{1}\right), v_{1}\left(x_{2}\right)\right\},\right. \\
\min \left\{\mu_{2}\left(x_{1}\right), v_{2}\left(x_{2}\right)\right\} \\
\ldots \min \left\{\mu_{k}\left(x_{1}\right), v_{k}\left(x_{2}\right)\right\} \\
\geq\left\{\operatorname { m i n } \left\{\min \left\{\mu_{1}(0), v_{1}(0)\right\},\right.\right. \\
\min \left\{\mu_{2}(0), v_{2}(0)\right\} \\
\left.\ldots \min \left\{\mu_{k}(0), v_{k}(0)\right\}\right\} \\
=(A \times B)(0,0)
\end{gathered}
$$

This implies that $(A \times B)\left(x_{1}, x_{2}\right)>(A \times B)(0,0)$, which is a contradiction that $A \times B$ is a multi-fuzzy BG-ideal of $X \times X$. Therefore either $A(0) \geq A(x)$ or $B(0) \geq B(x)$.

Let $B(0)<A(x)$ and $B(0)<B(x)$. Then $v_{i}(0)<\mu_{i}(x)$ and $v_{i}(0)<v_{i}(x) \forall i=1,2, \ldots k$

$$
\begin{array}{r}
(A \times B)(0,0)=\left\{\min \left\{\mu_{1}(0), v_{1}(0)\right\},\right. \\
\min \left\{\mu_{2}(0), v_{2}(0)\right\} \ldots \min \left\{\mu_{k}(0), v_{k}(0)\right\} \\
=\left\{v_{1}(0), v_{2}(0), \ldots v_{k}(0)\right\} \\
=B(0) \\
(A \times B)\left(x_{1}, x_{2}\right)=\left\{\min \left\{\mu_{1}\left(x_{1}\right), v_{1}\left(x_{2}\right)\right\},\right. \\
\min \left\{\mu_{2}(0), v_{2}(0)\right\} \ldots \min \left\{\mu_{k}(0), v_{k}(0)\right\} \\
\geq\left\{v_{1}(0), v_{2}(0), \ldots v_{k}(0)\right\} \\
=B(0) \\
=(A \times B)(0,0)
\end{array}
$$

This implies that $(A \times B)\left(x_{1}, x_{2}\right)>(A \times B)(0,0)$, which is a contradiction that $A \times B$ is a multi-fuzzy BG-ideal of $X \times$ $X$. Therefore either $A(0) \geq A(x)$ then either $B(0) \geq A(x)$ or $B(0) \geq B(x)$.

The third proof is similar to second proof.

Theorem 4.4. If $A \times B$ is a multi-fuzzy $B G$-ideal of $X \times X$, then $A$ or $B$ is a multi-fuzzy $B G$-ideal of $X$.

Proof. To prove that $B$ is a multi-fuzzy BG-ideal of $\mathrm{X}$. By theorem 4.3(i), either $A(0) \geq A(x)$ or $B(0) \geq B(x)$. Assume that $B(0) \geq B(x)$. Also from theorem 4.3(iii), either $A(0) \geq A(x)$ or $A(0) \geq B(x)$. If $A(0) \geq B(x)$, then $\mu_{i}(0) \geq$ $v_{i}(x) \forall i=1,2, \ldots k$.

$$
\begin{aligned}
(A \times B)(0, x)=\{ & \min \left\{\mu_{1}(0), v_{1}(x)\right\}, \\
& \min \left\{\mu_{2}(0), v_{2}(x)\right\} \ldots \\
& \left.\min \left\{\mu_{k}(0), v_{k}(x)\right\}\right\} \\
= & \left\{v_{1}(x), v_{2}(x), \ldots v_{k}(x)\right\} \\
= & B(x)
\end{aligned}
$$




$$
\begin{aligned}
B(x)= & (A \times B)(0, x) \\
\geq & \min \{(A \times B)((0, x) *(0, y)), \\
& (A \times B)(0, y)\} \\
= & \min \{(A \times B)((0 * 0),(x * y)), \\
& (A \times B)(0, y)\} \\
= & \min \{B(x * y), B(y)\}
\end{aligned}
$$

$$
\begin{aligned}
\operatorname{Also} B(x * y) & =(A \times B)(0, x * y) \\
& =(A \times B)(0 * 0, x * y) \\
& =(A \times B)((0, x) *(0, y)) \\
& \geq \min \{(A \times B)(0, x),(A \times B)(0, y)\} \\
& =\min \{B(x), B(y)\}
\end{aligned}
$$

Hence $B$ is a multi-fuzzy BG-ideal of $X$.

To prove that $A$ is a multi-fuzzy BG-ideal of $\mathrm{X}$.

By theorem 4.3(i), either $A(0) \geq A(x)$ or $B(0) \geq B(x)$. Assume that $A(0) \geq A(x)$. Also from theorem 4.3(ii), either $B(0) \geq A(x)$ or $B(0) \geq B(x)$.

If $B(0) \geq A(x)$, then $v_{i}(0) \geq \mu_{i}(x) \forall i=1,2, \ldots k$.

$$
\begin{aligned}
&(A \times B)(x, 0)=\{ \min \left\{\mu_{1}(x), v_{1}(0)\right\}, \\
& \min \left\{\mu_{2}(x), v_{2}(0)\right\} \ldots \\
&\left.\min \left\{\mu_{k}(x), v_{k}(0)\right\}\right\} \\
&=\left\{\mu_{1}(x), \mu_{2}(x), \ldots \mu_{k}(x)\right\}=A(x) \\
& A(x)=(A \times B)(x, 0) \\
& \geq \min \{(A \times B)((x, 0) *(y, 0)),(A \times B)(y, 0)\} \\
&= \min \{(A \times B)((x * y),(0 * 0)), \\
&(A \times B)(y, 0)\} \\
&= \min \{A(x * y), A(y)\}
\end{aligned}
$$

$$
\begin{aligned}
\operatorname{Also} A(x * y) & =(A \times B)(x * y, 0) \\
& =(A \times B)(x * y, 0 * 0) \\
& =(A \times B)((x, 0) *(y, 0)) \\
& \geq \min \{(A \times B)(x, 0),(A \times B)(y, 0)\} \\
& =\min \{A(x), A(y)\}
\end{aligned}
$$

Hence $A$ is a multi-fuzzy BG-ideal of $X$.

Theorem 4.5. If $A$ and $B$ are two normal multi-fuzzy $B G$ ideals of $X$, then $A \times B$ is a normal multi-fuzzy $B G$-ideal of $X \times X$.

Proof. Let $A$ and $B$ be two normal multi-fuzzy BG-ideals of $X$. Then $A(0)=1_{k}$ and $B(0)=1_{k}$. This implies that $\mu_{i}(0)=$ $1, v_{i}(0)=1$ fori $=1,2, \ldots k$.

$(A \times B)(0,0)=\left\{\min \left\{\mu_{1}(0), v_{1}(0)\right\}, \min \left\{\mu_{2}(0), v_{2}(0)\right\} \ldots\right.$ $\left.\min \left\{\mu_{k}(0), v_{k}(0)\right\}\right\}=\{\min \{\{1,1\}, \min \{1,1\} \ldots \min \{1,1\}\}=$ $(1,1,1 \ldots$ ktimes $)=1_{k}$

Hence $A \times B$ is a normal multi-fuzzy BG-ideal of $X \times X$.

\section{Conclusion}

The notion of normal multi-fuzzy BG-ideals of BG-algebra is introduced by applying the concept of multi-fuzzy sets in ideals of BG-algebra and its properties are studied. Also the cartesian product of multi-fuzzy BG-ideals of BG-algebra is defined and its properties are discussed.

\section{References}

[1] M.Abu Ayub Ansari, M.Chandramouleeswaran, Normal fuzzy $\beta$-subalgebras of $\beta$-algebras, Applied Mathematical Sciences, 7(105)(2013), 5213-5224.

[2] S.S. Ahn and K.S.So, On (Complete) Normality of Fuzzy d-ideals in d-algebrasScientiae Mathematicae japonicae Online,(2008),453-460.

[3] S.S.Ahn, H.D.Lee, Fuzzy Subalgebras of Bg-algebras, Commun.Korean Math.Soc.,,19(2)(2004), 243-251.

[4] Y.Imai, K.Iseki, On axiom system of propositional calculi XIV, Proc.Japan Academy 42((1966)), 19-22.

[5] K.Iseki, S.Tanaka, An introduction to theory of BCKalgebras, Math.Japonica, 23((1978), 1-26.

[6] K.Iseki, On BCI-algebras, Math.Seminar Notes,8(1980), 125-130.

[7] C.B.Kim, H.S.Kim, On BG-algebras, Demonstration Mathematica, 41(2008), 497-505.

[8] R.Muthuraj, M.Sridharan \& P.M.Sitharselvam, Fuzzy BG-ideals in BG-algebra, International Journal of Computer Applications, 2(1)(2010), 26-30.

[9] R.Muthuraj, S.Devi,,Multi-fuzzy Subalgebras of BGalgebra and Its Level Subalgebras,International Journal of Applied Mathematical Sciences, 9(1)(2016), 113-120.

[10] R.Muthuraj, S.Devi, A Study on Intuitionistic MultiFuzzy Ideals of BG-algebra Middle-East Journal of Scientific Research, 25(2) (2017),335-340.

[11] R.Muthuraj, S.Devi, Multi Anti-Fuzzy Subalgebras and Homomorphism of Multi-fuzzy Subalgebras of BGalgebraInternational Journal of Engineering Associates, 5(9) (2016), 15-18.

${ }^{[12]}$ R.Muthuraj, S.Balamurugan, Multi-Fuzzy Group and its Level Subgroups Gen.Math.Notes, 17(1) (2013), 74-81.

[13] R.Muthuraj, S.Devi, Multi-fuzzy BG-ideals in BGalgebra Annals of Pure and Applied Mathematics, 15(2)(2017), 193-200.

[14] J.Neggers,H.S.Kim, On B-algebras, Mat.Vesnik,54(2002), 21-29.

[15] Dr.A.Prasanna, M.Premkumar, Hajee.Dr.S.Ismail Mohideen, Normalization of fuzzy BG-ideals in BG-algebra International Journal of Mathematics Trends and Technology,53(4) (2018), 270-276.

[16] Dr.A.Prasanna, M.Premkumar, Hajee.Dr.A.Solairaju, Normalization of fuzzy B-ideals in B-algebra International Journal of Mathematics Trends and Technology,53(4) (2018), 277-283.

[17] S.Sabu, T.V.Ramakrishnan, Multi-fuzzy sets, International Mathematical Forum,50(2010), 2471-2476. 
[18] S.Sabu,T.V.Ramakrishnan Multi-fuzzy Topology, International Journal of Applied Mathematics, 24(1)(2011), 117-129.

[19] Wiestaw A.Dudek and Young Bae Jun,Normalizations of Fuzzy BCC-Ideals in BCC-algebrasMathematica Moravica, 3(1999), 17-24.

[20] L.A.Zadeh, Fuzzy Sets,it Information and control,8(1965),338-353.

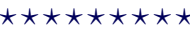

ISSN(P):2319-3786

Malaya Journal of Matematik

ISSN(O):2321 - 5666

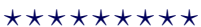

